\title{
Bioprocess engineering of microalgae to produce a variety of consumer products
}

\begin{abstract}
Microalgae biotechnology has recently emerged into the lime light owing to numerous consumer products that can be harnessed from microalgae. Product portfolio stretches from straightforward biomass production for food and animal feed to valuable products extracted from microalgal biomass, including triglycerides which can be converted into biodiesel. For most of these applications, the production process is moderately economically viable and the market is developing. Considering the enormous biodiversity of microalgae and recent developments in genetic and metabolic engineering, this group of organisms represents one of the most promising sources for new products and applications. With the development of detailed culture and screening techniques, microalgal biotechnology can meet the high demands of food, energy and pharmaceutical industries. This review article discusses the technology and production platforms for development and creation of different valuable consumer products from microalgal biomass.
\end{abstract}

Keyword: Microalgae; Cultivation techniques; Dewatering; Biofuels; Consumer products 